\title{
Screening for Cerebellopontine Angle Tumours: Conventional MRI vs T2 Fast Spin Echo MRI
}

\author{
Joseph C. Dort, David Sadler, William Hu, Carla Wallace, Pierre La Forge, \\ Robert Sevick
}

\begin{abstract}
Background: Unilateral audiovestibular symptoms are commonly seen in clinical practice and are rarely caused by retrocochlear pathology. However, clinicians are often required to rule out potentially serious causes of these unilateral symptoms. Gadolinium enhanced magnetic resonance imaging (GdMRI) is the most accurate test for detecting small cerebellopontine angle lesions and also screens the adjacent CNS structures. Its main disadvantage is the cost of the procedure. Methods: We studied 100 consecutive patients with both GdMRI and a newer MRI screening study utilizing unenhanced T2-weighted fast spin echo (fse) MRI. Acquired images were randomly assessed by a panel of three neuro-radiologists. Results: We found that the screening (fse) MRI was as sensitive and specific when detecting cerebellopontine angle tumors. Conclusions: We conclude that T2-weighted fse MRI is a safe and cost-effective alternative to GdMRI and offers better diagnostic utility when compared to auditory brain stem response (ABR) and CT scans.
\end{abstract}

\begin{abstract}
RÉSUMÉ: Dépistage des tumeurs de l'angle pontocérébelleux: comparaison de la RMN conventionnelle et de la RMN écho de spin rapide T2. Introduction: Des symptômes audiovestibulaires unilatéraux sont fréquemment observés en pratique clinique et sont rarement causés par une pathologie rétrocochléaire. Cependant, les cliniciens doivent souvent éliminer la possibilité que la cause de ces symptômes unilatéraux ne soit sérieuse. L'imagerie par résonance magnétique avec rehaussement par le gadolinium (GD-RMN) est l'examen le plus précis pour détecter les petites lésions de l'angle pontocérébelleux et également pour visionner les structures adjacentes du SNC. Son principal inconvénient est son coût. Méthodes: Nous avons étudié 100 patients consécutifs au moyen de la GD-RMN et une nouvelle technique de dépistage par RMN, la RMN écho de spin rapide T2 sans rehaussement (ésr). Les images acquises ont été évaluées au hasard par un groupe de trois neuroradiologistes. Résultats: Nous avons constaté que le dépistage par RMN (ésr) était aussi sensible et spécifique pour détecter les tumeurs de l'angle pontocérébelleux. Conclusions: Nous concluons que la RMN (ésr) est une alternative sûre et économique à la GDRMN et comporte des avantages diagnostiques par rapport aux potentiels évoqués auditifs du tronc cérébral et à la tomodensitométrie cérébrale.
\end{abstract}

Can. J. Neurol. Sci. 2001; 28: 47-50

Asymmetrical audiovestibular symptoms are common and when present often lead to an expensive series of investigations. These test batteries usually culminate with magnetic resonance imaging (MRI) as the definitive step in the elimination of significant retrocochlear disease. Although MRI is considered the "gold standard"1,2 for diagnosis, it is expensive and not infallible. ${ }^{3}$ At the Foothills Hospital, Calgary, we perform between 350 - 400 contrast enhanced MRI scans per year for patients with unilateral audiovestibular symptoms. The contrast agent alone costs $\$ 150$ per study and a physician must be present if contrast is being administered. This requirement limits the times during which images can be acquired.

A rapid, accurate and inexpensive imaging study would be very useful for investigating this large patient population.
Authors have modified standard MRI protocols in an effort to define screening algorithms that would meet these criteria. Harnsberger and his colleagues ${ }^{4,5}$ described a T2 fast spin echo (fse) technique that yielded high resolution images of the internal auditory canal (IAC), inner ear and cerebellopontine angle (CPA). These authors used a three inch phased-array temporo-

From the Departments of Surgery (Otolaryngology) (JCD), Radiology (DS, WH, CW, PLF, RS), and Clinical Neurosciences (JCD, DS, WH, CW, PLF, RS) Seaman Family Magnetic Resonance Research Centre (JCD, WH, CW, RS), University of Calgary, Calgary, Alberta, Canada.

Received March 28, 2000. Accepted in final Form SePtember 26, 2000. Reprint requests to: J.C. Dort, 3330 Hospital Dr. N.W., Calgary, AB, Canada T2N 4N1 
mandibular joint receiver coil to improve image quality. Stuckey et $\mathrm{al}^{6}$ also described an unenhanced T2 weighted MRI algorithm for effective screening in an even larger group of patients with suspected retrocochlear disease. Stuckey used standard head coils for this group of patients. These authors reported an accuracy of between 94 and 98 percent for the screening MRI study.

The current standard of care in Canada is to use T1-weighted, gadolinium enhanced MRI (T1/GdMRI) as the definitive test to rule out CPA tumors and other retrocochlear disease processes. We were interested in studying the effectiveness of a screening imaging protocol based on utilizing the T2-fse techniques alluded to above. In order to assess the accuracy of T2-fseMRI (T2/fseMRI), all patients underwent GdMRI as the "gold standard".

\section{Methods}

This study was performed prospectively on a cohort of 100 consecutive patients referred to the Foothills Medical Centre MRI facility for investigation of unilateral audiovestibular symptoms or other symptoms suspicious of CPA tumor. Some of the patients had known tumors that were being followed with serial MRI. All patients underwent unenhanced T2 weighted fseMRI and also conventional T1 spin-echo GdMRI. Total tumor volume, including the intracanalicular portion, was measured in the greatest axial dimensions on an off-line workstation. Audiometric data were not collected for this study.

MRI covering the CPA was performed on a 1.5T (Horizon HiSpeed, GE) magnet. Each patient was scanned using a quadrature head coil. In addition to standard anatomical images of the brain, two high resolution sequences were performed.

1. Axial T2-weighted fse: 10 slices, $2 \mathrm{~mm}$ skip $0, \mathrm{TE}=105$, $\mathrm{TR}=6200, \mathrm{ETL}=64, \mathrm{FOV}=16 \times 12 \mathrm{~mm}$, Matrix $=512 \times 256$, $\mathrm{NEX}=4$.

2. Axial T1-weighted FSPGR post Gd-DTPA with fat saturation: 10 slices, $2 \mathrm{~mm}$ skip $0, \mathrm{TE}=4.2, \mathrm{TR}=250, \mathrm{FA}=90^{\circ}$, $\mathrm{FOV}=18 \times 18 \mathrm{~mm}$, Matrix=512 x 256, NEX=4.

The images were then reviewed by three neuroradiologists who compared both the conventional and fse images in random

Table 1: Sensitivity and Specificity (all lesions)

\begin{tabular}{lllll}
\hline & \multicolumn{4}{c}{ T1W / Gd } \\
T2W / fse & $-\mathrm{ve}$ & 542 & 6 & Sens $=89 \%$ \\
& $+\mathrm{ve}$ & 1 & 51 & Spec $=99.8 \%$ \\
\hline
\end{tabular}

Table 2: Sensitivity and Specificity (postsurgical follow-up patients excluded)

\begin{tabular}{lllll}
\hline & \multicolumn{4}{c}{ T1W / Gd } \\
T2W / fse & -ve & + ve & \\
& -ve & 522 & 1 & Sens $=96 \%$ \\
& + ve & 0 & 26 & Spec $=100 \%$ \\
\hline
\end{tabular}

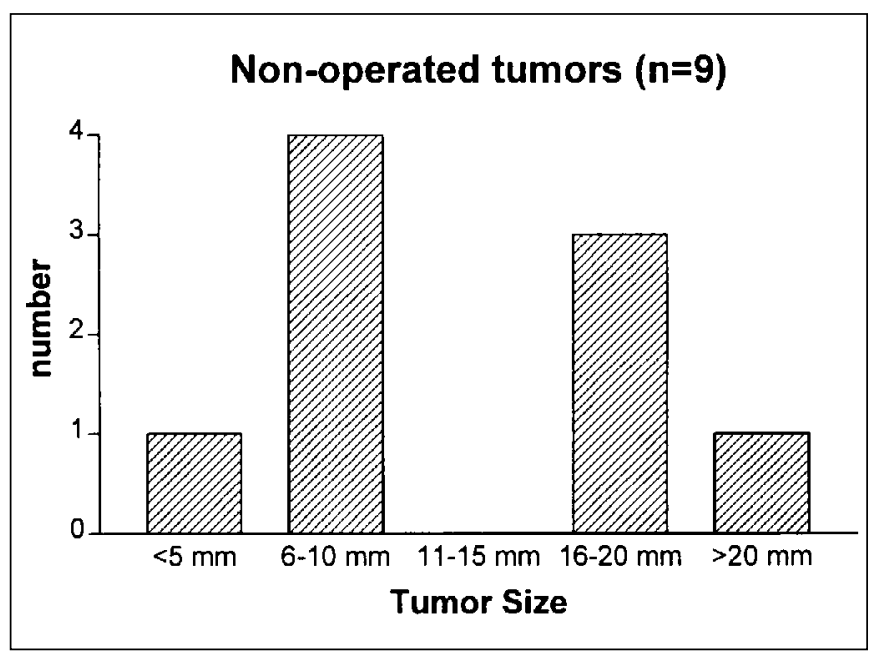

Figure 1: Tumor size distribution in the nonoperated lesions. The ten post surgical follow-up patients are excluded.

order. All radiologists examined all images in batches of 25 in order to avoid reader fatigue. The radiologists then rated the images for clarity and diagnostic quality and recorded their impressions. The data were then collected and analyzed. The radiologists were blinded to the nature and side of the patient's symptoms. The T1/GdMRI is believed to be the gold standard for diagnosis and was therefore assumed to have a sensitivity and specificity of $100 \%$. Although some patients had surgery, all patients with positive MRI scans did not undergo operation (the real gold standard).

\section{RESULTS}

\section{Prospective data}

Of the 100 patients, 51 were male and 49 were female ranging in age from 10 to 83 years. Fourteen patients with known tumors (one bilateral) were being followed with serial MRI and four new tumors were diagnosed for a total of 19 lesions in the study population. Internal auditory canal diameter was measured bilaterally and a mean IAC diameter of $4.6 \mathrm{~mm}$ (range $2-10$ $\mathrm{mm}$ ) was found.

Tumors of all sizes were found (Figure 1) and the one false negative fse study was in a patient with a $4 \mathrm{~mm}$ tumor. Sensitivity and specificity of the fse study is shown in Tables 1 and 2. Both sensitivity and specificity were higher in the nonoperated patients, suggesting that the fse imaging study was less accurate for postsurgical follow-up. As seen in Table 2, there was one false negative study with the T2-fse technique when we excluded the postoperative follow-up group of patients.

\section{Imaging example}

Figure 2 illustrates an example of the exquisite sensitivity offered by the T2/fse imaging algorithm. In this example, a patient presenting with unilateral auditory symptoms underwent MRI with both T2/fse and T1/Gd techniques. This patient has a small intracanalicular lesion that is clearly seen on the T2weighted fse study (Figure 2A) and the T1-Gd study (Figure 2B). 

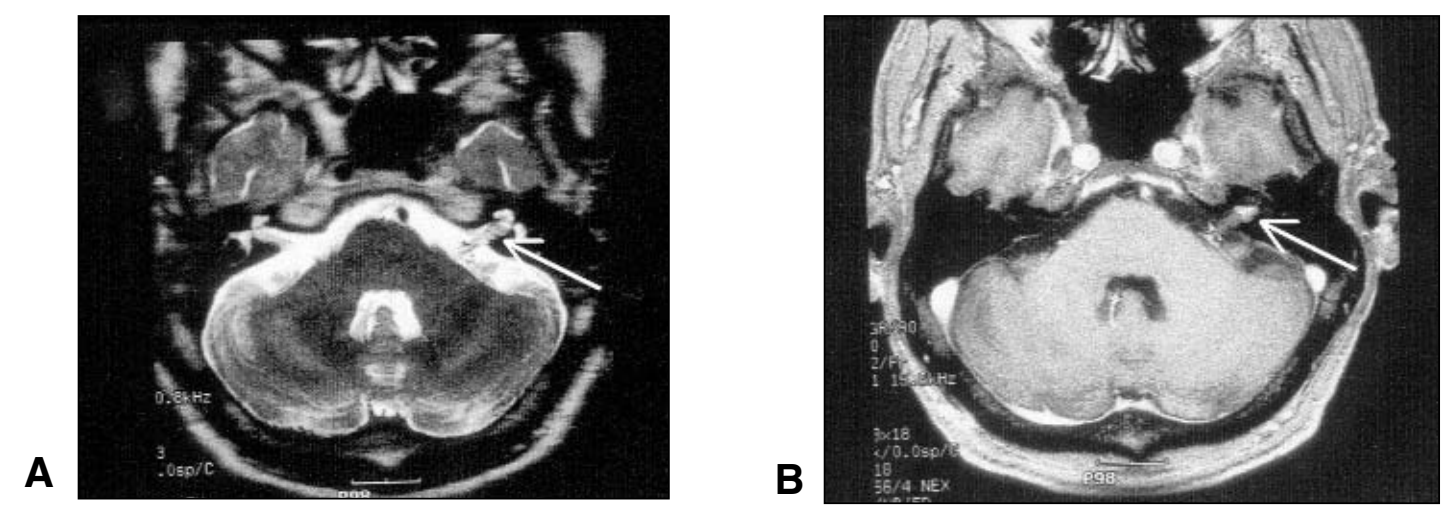

Figure 2: Comparison of T2/fse (A) and T1/Gd (B) images in a patient with a small, left-side intracanalicular lesion. The arrows indicate the lesion.

\section{Cost/time analysis}

It was not our intention to perform a formal and detailed cost analysis. Nevertheless our data lends itself to several observations. A standard Gd-enhanced posterior fossa study takes approximately nine minutes to complete (five minutes for contrast injection and four minutes for imaging). The T2-fse study takes approximately 2.5 minutes. In Calgary we perform about 400 MR studies per year to evaluate patients with unilateral audiovestibular symptoms. Employing the T2-fse protocol uses about 16 hours of imaging time as compared to 60 hours of imaging time if the Gd-enhanced protocol is used. Using the T2-fse study would result in approximately $\$ 60,000.00$ savings for contrast alone (400 patients x $\$ 150$ / study).

\section{Discussion}

The search for better diagnostic algorithms in patients with unilateral audiovestibular symptoms is ongoing. ${ }^{7,8}$ Newer and better technology is often more expensive thereby leading to limited access. GdMRI is the most accurate noninvasive method of diagnosing a CPA tumor. ${ }^{8-10}$ However, some authors ${ }^{11}$ believe that auditory brain stem response (ABR) and other auditory tests still have an important role to play in the diagnostic work-up of patients with unilateral audiovestibular symptoms. Therefore, significant controversy remains regarding the best method of investigating patients.

Although vestibular schwannomas are uncommon, ${ }^{12}$ unilateral audiovestibular symptoms are not. ${ }^{1}$ In Canada, and increasingly in the United States, it is not possible to get GdMRI for all patients with unilateral audiovestibular symptoms where vestibular schwannoma is a diagnostic consideration. In recognition of these cost constraints, investigators have developed less costly imaging algorithms.

The use of $\mathrm{T} 2$-weighted (T2W) fse images as a screening algorithm was initially proposed by radiologists in the United Kingdom ${ }^{13,14}$ and has subsequently been endorsed and applied by other authors. Allen, Harnsberger and colleagues ${ }^{15}$ used a noncontrast enhanced MRI study to diagnose patients with CPA lesions. These authors found that the noncontrast study was significantly faster and less expensive than a standard GdMRI and the screening study was not less accurate in their hands. Subsequent work from the same group reveals a $54 \%$ reduction in screening costs using the fse imaging algorithms. ${ }^{16}$ Since these initial reports, others have shown similar results; namely high diagnostic yield and lower cost when fseMRI is used as a screening test. ${ }^{17,18}$ In our study the neuroradiologists had no diagnostic information and they prospectively compared both conventional T1 weighted GdMRI with the fse images. However, the images were presented randomly in order to avoid interpretation bias.

Despite excellent results with noncontrast enhanced MRI there is no universal agreement about the role of these screening examinations. Jackler, in a 1996 editorial, raises the possibility of missing very small lesions with the fse study ${ }^{19}$ and it has been stated that these very small lesions are most amenable to treatment with the least morbidity.

Wilson and colleagues describe a screening GdMRI technique that potentially overcomes the limitations of fseMRI. ${ }^{20}$ These authors use T2-fse images combined with T1-weighted sections using a half-dose of gadolinium. Their total imaging time is about eight minutes and the examination costs about one third as much as a standard MRI. Wilson's data suggest that diagnostic accuracy is not compromised and that this protocol addresses the criticisms raised by others. ${ }^{19}$

In the past, $\mathrm{ABR}$ has played a role in screening and diagnosis of retrocochlear hearing loss. The role of ABR has become less clear when one considers tumors smaller than $1 \mathrm{~cm}$ in size: ABR will miss as many as $17 \%$ of these lesions. ${ }^{7}$ A newer, more sensitive, ABR method has been developed by Don and colleagues..$^{21}$ These authors, using the stacked derived-band ABR technique, demonstrated the ability to detect intracanalicular acoustic schwannomas that were missed by conventional ABR methods. This method has not yet been widely applied but it may prove to be a cost-effective means of selecting patients for imaging studies.

Where does this data leave us? The ideal test is inexpensive, noninvasive and $100 \%$ sensitive and specific. In our hands the T2/fse MRI study nearly achieves these goals. The screening GdMRI described by Wilson may be another alternative. Our experience in managing patients with acoustic schwannomas is 
similar to others in that patients with small tumors may present with very subtle symptoms and diagnosis is often challenging. A negative ABR or screening MRI may offer false reassurance that nothing is wrong and the opportunity to treat the patient with the least risk is lost. ${ }^{19}$ At the present time we have patients with unilateral audiovestibular symptoms and a negative screening MRI return for a follow-up clinical examination and complete auditory assessment after one year. We also emphasize to these patients that although a negative imaging study is reassuring there is still a small but real possibility that a tiny lesion was missed. Our hope for the future is that new technologies, such as stacked ABR, will lead to the elusive "perfect" screening test.

\section{Conclusions}

The current study shows that T2/fse MRI is an accurate method of diagnosing patients with lesions involving the CPA and IAC. It is not useful for following patients after surgery nor does it screen for other causes of unilateral audiovestibular symptoms. T2-fse MRI is now an important part of our imaging armamentarium in this selected patient population.

\section{ACKNOWLEDGEMENTS}

Dr Dort acknowledges the support of the Campbell McLaurin Foundation for Hearing Deficiencies

\section{REFERENCES}

1. Raber E, Dort JC, Sevick R, Winkelaar R. Asymmetric hearing loss: toward cost-effective diagnosis. J Otolaryngo 1997; 26(2):88-91.

2. Robson AK, Leighton SE, Anslow P, Milford CA. MRI as a single screening procedure for acoustic neuroma: a cost effective protocol [see comments]. J R Soc Med 1993; 86(8):455-457.

3. Han MH, Jabour BA, Andrews JC, et al. Nonneoplastic enhancing lesions mimicking intracanalicular acoustic neuroma on gadolinium-enhanced MR images. Radiology 1991; 179:795-796.

4. Allen RW, Harnsberger HR, Shelton C, et al. Low-cost highresolution fast spin echo MR of acoustic schwannoma: an alternative to enhanced conventional spin-echo MR? Am J Neuroradiol 1996; 17:1205-1210.

5. Shelton C, Harnsberger HR, Allen R, King B. Fast spin echo magnetic resonance imaging: clinical application in screening for acoustic neuroma. Otolaryngol Head Neck Surg 1996; 114(1):71-76.
6. Stuckey SL, Harris AJ, Mannolini SM. Detection of acoustic schwannoma: use of constructive interference in the steady state three-dimensional MR. Am J Neuroradiol 1996; 17:1219-1225.

7. Gordon ML, Cohen NL. Efficacy of auditory brainstem response as a screening test for small acoustic neuromas. Am J Otol 1995; 16(2):136-139.

8. House JL, Tubergen LB, Burt M. Current diagnosis of acoustic tumors. Indiana Med 1989; 89(9):706-709.

9. Hashimoto S, Kawase T, Furukawa K, Takasaka T. Strategy for the diagnosis of small acoustic neuromas. Acta Otolaryngol Suppl 1991; 91;481:567.

10. Welling DB, Glasscock ME, Woods CI, Jackson CG. Acoustic neuroma: a cost-effective approach. Otolaryngol Head Neck Surg 1990; 103:364-370.

11. Frazer GJ. Auditory brainstem response audiometry and acoustic neuromas. Am J Otol 1997; 18(1):126-128.

12. Frohlich AM, Sutherland GR. Epidemiology and clinical features of vestibular schwannoma in Manitoba, Canada. Can J Neurol Sci 1993; 20(2):126-130.

13. Phelps PD. Fast spin echo MRI in otology. J Laryngol Otol 1994; 108(5):383-394

14. Renowden SA, Anslow P. The effective use of magnetic resonance imaging in the diagnosis of acoustic neuromas. Clin Radiol 1993; 48(1):25-28.

15. Allen RW, Harnsberger HR, Shelton C, et al. Low-cost highresolution fast spin echo MR of acoustic schwannoma: an alternative to enhanced conventional spin-echo MR? Am J Neuroradiol 1996; 17:1205-1210.

16. Daniels RL, Shelton C, Harnsberger HR. Ultra high resolution nonenhanced fast spin echo magnetic resonance imaging: costeffective screening for acoustic neuroma in patients with sudden sensorineural hearing loss. Otolaryngol Head Neck Surg 1998; 119(4):364-369.

17. Soulie D, Cordoliani YS, Vignaud J, Cosnard G. MR imaging of acoustic neuroma with high resolution fast spin echo T2weighted sequence. Eur J Radiol 1997; 24(1):61-65.

18. Tan TY. Noncontrast high resolution fast spin echo magnetic resonance imaging of acoustic schwannoma. Singapore Med J 1999; 40(1):27-31.

19. Jackler RK. Cost-effective screening for acoustic neuroma with unenhanced MR: a clinician's perspective. Am J Neuroradiol 1996; 17:1226-1228.

20. Wilson DF, Talbot JM, Mills L. A critical appraisal of the role of auditory brain stem response and magnetic resonance imaging in acoustic neuroma diagnosis. Am J Otol 1997; 18(5):673-681.

21. Don M, Masuda A, Nelson R, Brackmann D. Successful detection of small acoustic tumors using the stacked derived-band auditory brain stem response amplitude. Am J Otol 1997; 18(5):608-621. 